\title{
Fundamental limits of diversity coherent reception on atmospheric optical channels
}

\author{
Aniceto Belmonte \\ Technical University of Catalonia, Department of Signal Theory and Communications, 08034 Barcelona, Spain \\ belmonte@tsc.upc.edu \\ Joseph M. Kahn \\ Stanford University, Department of Electrical Engineering, Stanford, CA 94305, USA
}

\begin{abstract}
We study the performance of diversity combining techniques applied to synchronous laser communication through the turbulent atmosphere. We consider the effects of log-normal amplitude fluctuations and Gaussian phase fluctuations, in addition to local oscillator shot noise. We study the effect of various parameters, including the ratio of receiver aperture diameter to wavefront coherence diameter, the scintillation index, and the number of independent diversity branches combined at the receiver. We consider both maximal-ratio combining (MRC) and selective combining (SC) diversity schemes. We derive expressions for the outage Shannon capacity, thus placing upper bounds on the spectral efficiency achievable using these techniques.
\end{abstract}

Keywords- Atmospheric turbulence; Optical communications; Coherent receivers; Diversity combining; Shannon capacity.

\section{INTRODUCTION}

In this work, we analyze the spectral efficiency of optical communication over the clear turbulent atmosphere. Defined as the average transmitted data rate per unit bandwidth for a specific average transmit power and probability of outage or error, achievable spectral efficiency is an important performance measure for free-space optical communication systems. As the use of multilevel phase modulation schemes increase spectral efficiency by sending multiple bits per symbol [1], optical coherent reception provides an alternative to direct detection schemes for free-space optical communication applications. In a coherent receiver, however, accurate wavefront matching between the incoming signal and the local oscillator is needed to ensure effective coherent reception. Imperfect spatial mode matching can lead to destructive interference and, consequently, to degraded system performance.

The presence of atmospheric turbulence complicates the problem, as the efficiency of a single-aperture coherent receiver is limited by the phase coherence length [2]. Atmospheric fading leads to serious degradation in the receiver sensitivity measured in terms of received photons per symbol, resulting in either a higher error rate or a higher required transmit power for a given multilevel modulation technique. A fading compensation technique that adapts the receiver to the channel fading can lead to better utilization of the channel capacity. This adaptive coherent receiver may be implemented by using phase aberration measurements and an adaptive local oscillator spatially matched by a deformable mirror to the field entering the diffraction-limited optical system. For typical turbulence conditions, large gains in achievable rate of reliable communication supported by the atmospheric channel are realizable by correcting a fairly small number of aberration modes and using optimum receiving aperture diameters.

This paper considers the analysis of coherent free-space optical diversity-combining channels corrupted by atmospheric turbulence from an information theory perspective. We analyze how these channels, by providing redundant replicas of the transmitted message to the receiver, each corrupted independently by the atmosphere, produce reliable communication because of the low probability of simultaneous deep fades in all the diversity channels. In focal- and pupilplane lenslets mosaic arrays, fading correlation can become important due to reduced element spacing. We have derived new statistical models for diversity receiver performance, considering various parameters, including the ratio of receiver aperture diameter to wavefront coherence diameter, the scintillation index, the effect of fading correlation, and the number of effective independent diversity branches combined at the receiver. These models are used to derive fundamental limits on outage probability and achievable rates for synchronous coherent links.

We have separately quantified the effects of amplitude fluctuations and phase distortion, and have identified the impact of the number of diversity branches on the maximal rate at which the information may be transferred. In most situations considered, phase distortion is the dominant performancelimiting effect, and amplitude fluctuations are of less importance. We have examined information-theoretic limits on the outage capacity, and have obtained simple analytical results. For typical turbulence conditions, sizeable gains in achievable rate are obtained using a rather small number of apertures. This study allows for the optimization of channel capacity over a variety of turbulence conditions and for the selection of maximum code rates at a given probability of outage. 


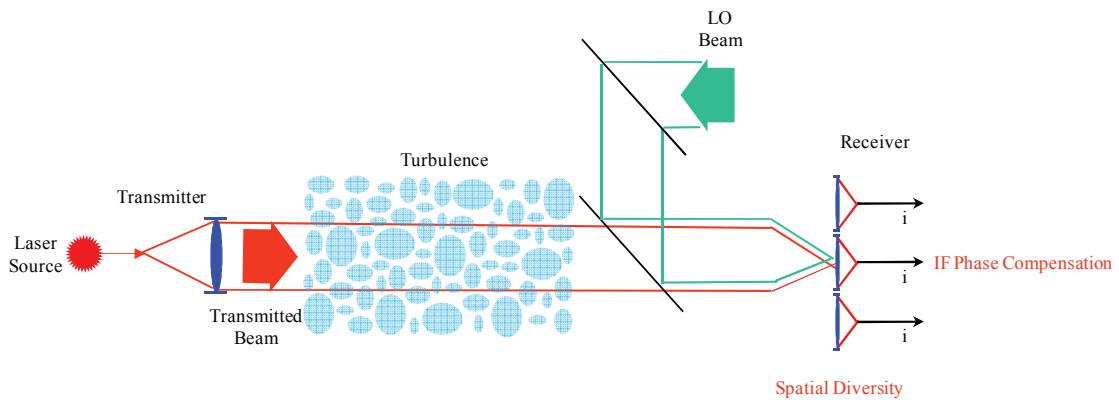

Fig. 1. A coherent free-space optical communication system is affected by the presence of atmospheric turbulence in many ways. First, turbulence induces a decrease of the mean received power level, which translates into a diminished signal-to-noise ratio in the receiver. Second, amplitude scintillation and phase distortion in the receiver plane act as intense sources of noise distorting the quality of the optical signal available for processing. All of them add together to deteriorate the overall communication performance of the optical systems. Coherent array receivers could be considered to alleviate the deteriorating effects of atmospheric turbulence in the performance of coherent receivers in free-space optical communications..

\section{OUTAGE PROBABILITY OF COHERENT ATMOSPHERIC LINKS USING DIVERSITY COMBINING}

As an alternative to a single monolithic-aperture coherent receiver with a full-size collecting area, a large effective aperture can be achieved by combining the output signal from an array of smaller receivers (see Fig. 1). The advantage of a coherent array in terms of the coupling efficiency is that the number of turbulence speckles over each subaperture in the array is much smaller than it would be over a single large aperture. Because each receiver can now be smaller than the scale on which the signal wavefront varies, the local oscillator phase can be matched to the signal to achieve effective coherent reception. Output signals from these receivers can then be combined electronically to improve the detection statistics. In general, the performance of a combined system should improve with an increasing number of receivers and, consequently, given a fixed collecting area, the combined system can offer superior performance. It is important to note that diversity combining consists of receiving redundantly the same information signal over two or more fading channels and to exploit the low probability of concurrence of deep fades in all the diversity channels. We define a general model for the output SNR of diversity systems over correlated fading channels. For systems where the atmospheric fading on the branches is correlated or dependent, we can solve the problem by transforming it into an independent problem using the technique of spatial whitening.

In free-space optical communication through the turbulent atmosphere, we must consider fading channels, which are a class of channels with multiplicative noise [3]. In the fading AWGN channel with average power constraint $P$ and noise power spectral density $N_{0} / 2$, we let $\alpha^{2}$ denote the atmospheric channel power fading and $\left(P / N_{0} B\right) \alpha^{2}=\gamma_{0} \alpha^{2}$ denote the instantaneous received SNR per symbol. For a shot-noiselimited coherent optical receiver, the SNR of the envelope detector can be taken as the number of signal photons detected on the receiver aperture $\gamma_{0}$ multiplied by a heterodyne mixing efficiency $\alpha^{2}$ : In addition to the effective delivery of the signal to the detector, the performance of the optical link also depends on the receiver sensitivity measured in terms of received photons. For systems with perfect spatial mode matching the heterodyne mixing efficiency is equal to 1 . When the spatial modes are not properly matched, the contribution to the current signal from different parts of the receiver aperture can interfere destructively and result in the reduced instantaneous heterodyne mixing and consequent fading.

We have already modeled the impact of atmospheric turbulence-induced phase and amplitude fluctuations on freespace optical links using synchronous detection and found that the SNR $\gamma$ for a single monolithic-aperture coherent receiver is described by a noncentral chi-square probability distribution function (PDF) with two degrees of freedom [4]. The model leading to the chi-square PDF is based on the observation that the downconverted signal current can be characterized as the sum of many contributions from $N$ different coherent regions within the aperture [4]. In this model, the signal is characterized as the sum of a constant (coherent) term and a random (incoherent) residual halo.

We consider two types of receive diversity combining [5]. First we assume the receiver has knowledge of the instantaneous channel state, making perfect maximal ratio combining (MRC) diversity possible. In this diversity scheme, the receiver co-phases the intermediate signals, adjusts their amplitudes separately, and sums them to obtain a composite signal with improved SNR. The rate at which phase and amplitude must be adjusted will be dictated by the rate at which the atmospheric turbulence fluctuates, generally no higher than $1 \mathrm{kHz}$. The MRC receiver is the optimal combining technique in that it yields a carrier with the highest mean SNR and lowest SNR fading. The optimum electronic gain for each receiver should be proportional to the received signal field 

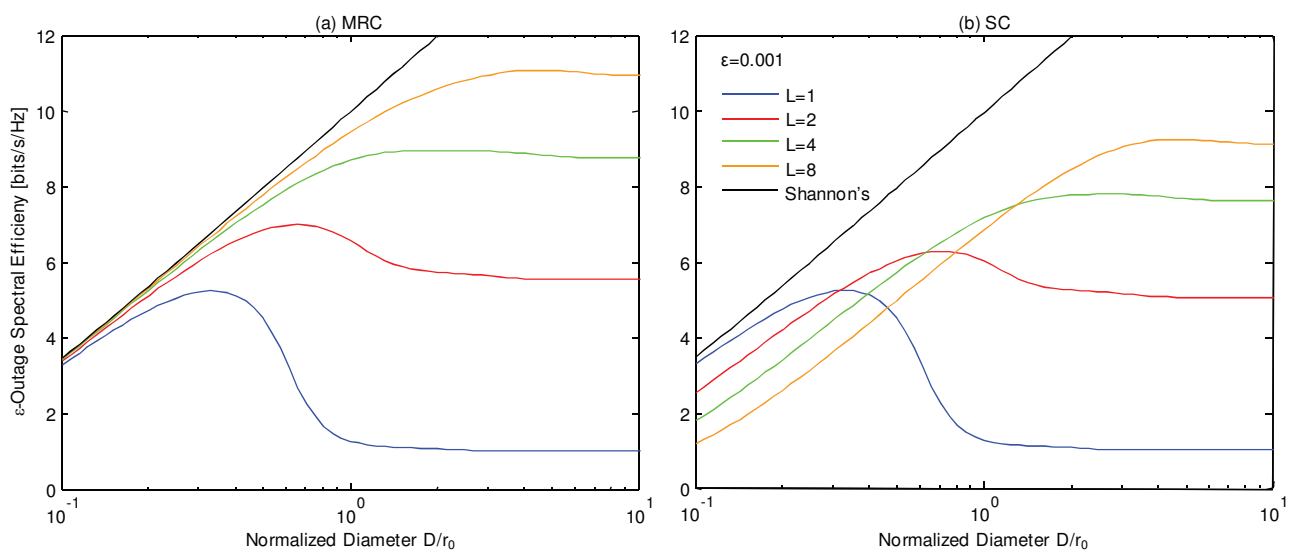

Fig. 2. $\varepsilon$-outage spectral efficiency vs. normalized receiver aperture diameter $D / r_{0}$ for coherent detection and AWGN. In (a), MRC combining is employed. In (b), a SC combiner is considered. In all cases, the outage probability is fixed at $\varepsilon=0.001$, and the channel capacity per unit bandwidth is shown for different values of the number $\mathrm{L}$ of combiner branches. The case $\mathrm{L}=1$ corresponds to no receive diversity (blue lines). The area $\pi \mathrm{D}^{2}$ describes the combined, multi-aperture system equivalent aperture. When no receive diversity is considered, D equals the receiver aperture diameter. The turbulence-free SNR per symbol $\gamma_{0}$ is proportional to the square of the aperture diameter $D$. For the smallest aperture considered, we assume $\gamma_{0}$ equal to 10 photons per symbol. In this plot, we neglect amplitude fluctuations by assuming $\sigma_{\beta}{ }^{2}=0$. In this case, turbulence is characterized by the phase coherence length $r_{0}$. The AWGN Shannon limit is indicated by black lines.

amplitude. Note that when the electronic gains and phase delays are back-propagated into the LO, the optimum gain and phase adjustments would result in an amplitude and phase match of this synthetic LO field to the distorted signal field. Then we consider the case when selective combining (SC) diversity schemes is used. A selection receiver is quite different from the coherent MRC summing receiver as the single strongest intermediate signal is switched to the output, while all the other array element signals are discarded. Although this simple diversity technique clearly does little to improve the mean SNR value of the collected signal, it does provide a reduction in the signal fading.

\section{OUTAGE CAPACITY}

In this work, we analyze the spectral efficiency of optical communication over the clear turbulent atmosphere. Defined as the average transmitted data rate per unit bandwidth for a specific average transmit power and probability of outage or error [1], achievable spectral efficiency is an important performance measure for free-space optical communication systems. As the use of multilevel phase modulation schemes increase spectral efficiency by sending multiple bits per symbol, optical coherent reception provides an alternative to direct detection schemes for free-space optical communication applications.

Figures 2-4 compare the spectral efficiency of an AWGN channel [6] with the spectral efficiency of a fading atmospheric channel with various diversity combining techniques [7]. Figure 2 considers the effect of aperture diameter on the $\varepsilon$ - outage spectral efficiency. It presents the spectral efficiency as a function of the normalized aperture $D / r_{0}$ for a constant phase coherence length $r_{0}$. In (a), MRC combining is employed. In (b), a SC combiner is considered. In all cases, the outage probability is small and fixed at $\varepsilon=0.001$, and the channel capacity per unit bandwidth is shown for different values of the number $L$ of combiner independent branches. The case $L=1$ corresponds to no receive diversity. The area $\pi D^{2}$ describes the combined, multi-aperture system equivalent aperture. When no receive diversity is considered, $D$ equals the receiver aperture diameter. If a $L$-aperture system is analyzed, each one of the aperture diameters equals $D / \sqrt{ } L$. For the smallest aperture considered, we assume $\gamma_{0}$ equal to 10 photons-per-symbol. For any other aperture diameter, the value of $\gamma_{0}$ is proportional to $D^{2}$. We study the outage channel capacity as a function of several parameters: the average signal-to-noise ratio (photons) per bit, the strength of atmospheric turbulence, the receiver aperture diameter $D$, and the number $\mathrm{L}$ of combiner branches. Turbulence effects are described by two parameters. Fried's coherence length $r_{0}$ describes the coherent diameter of the distorted wavefront phase. The scintillation index $\sigma_{\beta}$ describes the intensity of amplitude fluctuations. In our modeling, we have chosen typical values of these two parameters. Atmospheric fading leads to serious degradation in the receiver sensitivity measured in terms of received photons per symbol, resulting in either a higher error rate or a higher required transmit power for a given multilevel modulation technique. In general, the performance of an array signal-combining system should improve with an increasing number of receivers and, consequently, given a fixed collecting area, the combined system can offer superior performance. 

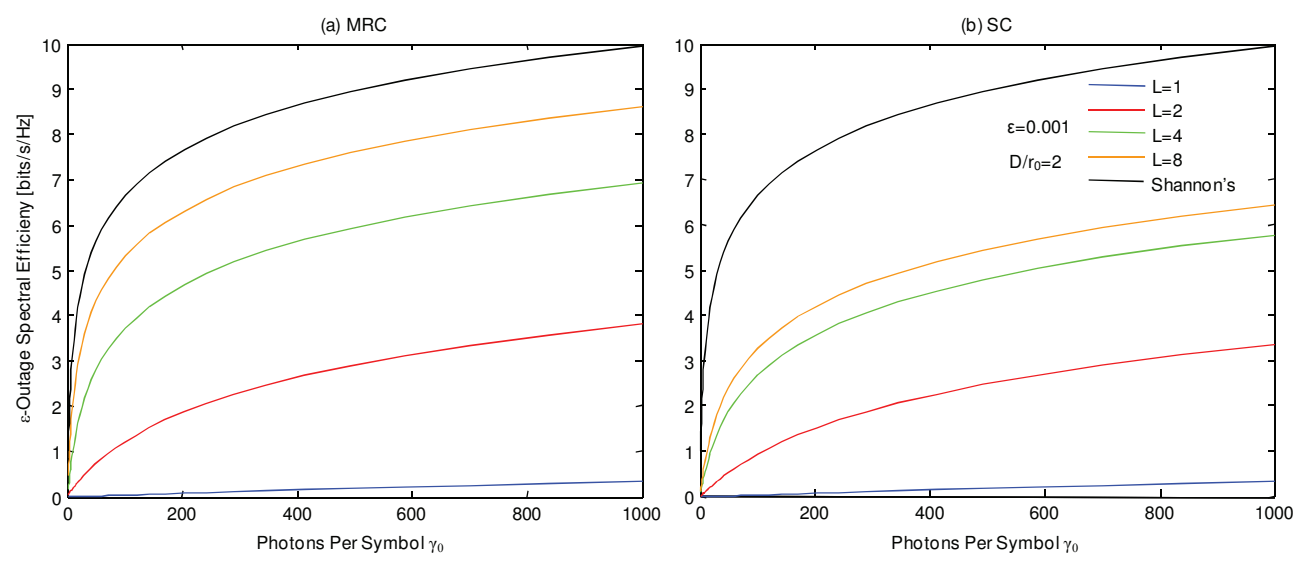

Fig. 3. $\varepsilon$-outage spectral efficiency vs. turbulence-free photons per symbol $\gamma_{0}$ for coherent detection and additive white Gaussian noise (AWGN). In (a), MRC combining is employed. In (b), a SC combiner is considered. In all cases, the outage probability is fixed at $\varepsilon=0.001$, and the channel capacity per unit bandwidth is shown for different values of the number $\mathrm{L}$ of combiner branches. The case $\mathrm{L}=1$ corresponds to no receive diversity (blue lines). Amplitude fluctuations are neglected by assuming $\sigma_{\beta}{ }^{2}=0$. Turbulence is characterized by a moderate phase coherence length $r_{0}$ such as $D / r_{0}=2$. The area $\pi \mathrm{D}^{2}$ describes the combined, multi-aperture system equivalent aperture. The AWGN Shannon limit is indicated by black lines.

Note the diminishing capacity returns that are obtained as the number of branches increases. Although as we increase L the outage spectral efficiency improves appreciably, the greatest improvement is still obtained in going from single- to two-branch combining. In any case, even for such small outage probability $\varepsilon=0.001$, a multiple receiver system with $\mathrm{L}=8$ apertures reach an outage spectral efficiency of over 10 bits/s/Hz, very close to the optimal spectral rate of a single branch AWGN channel as defined by Shannon [6]. When a single $(\mathrm{L}=1)$ or dual $(\mathrm{L}=2)$ receiver system is considered, the
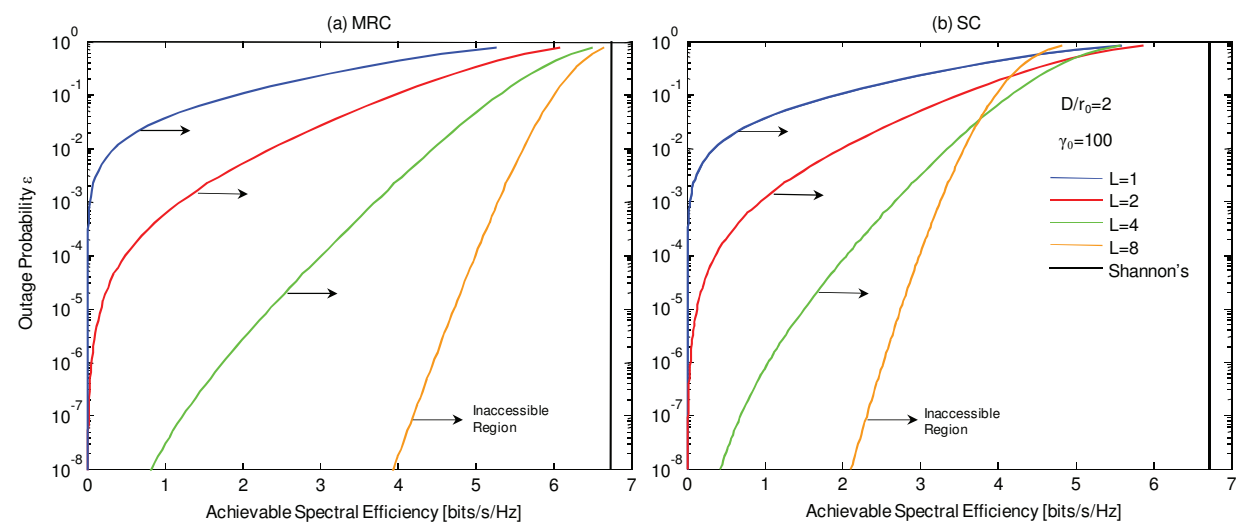

Fig. 4. Probability of outage versus channel capacity per unit bandwidth for coherent detection and additive white Gaussian noise (AWGN). We consider both MRC (in a) and SC (in b) combining of the received signal and the trade-off between the outage probability and the maximum achievable rate is analyzed for different values of the number of branches $\mathrm{L}$ in the combiner. The case $\mathrm{L}=1$ corresponds to no receive diversity (blue lines). In all cases, we assume the number of photons per symbol $\gamma_{0}$ equals to 100 . Amplitude fluctuations are neglected by assuming $\sigma_{\beta}{ }^{2}=0$. Turbulence is characterized by a moderate phase coherence length $r_{0}$ such that $D / r_{0}=2$. The area $\pi \mathrm{D}^{2}$ describes the combined, multi-aperture system equivalent aperture. The AWGN Shannon limit corresponding to $\gamma_{0}=100$ photons-per-symbol is indicated by vertical black lines. 
existence of an optimal aperture diameter in coherent freespace links is apparent. This optimal aperture diameter maximizes the $\varepsilon$-outage capacity. When the aperture is larger than the optimal value, phase distortion cannot be overcome by the increase in collected power, and we observe a decrease in capacity. For $\mathrm{L}>2$ receiver systems, or under strong scintillation conditions, no optimal value can be identified.

In Fig. 4, the tradeoff between the outage probability $\varepsilon$ and the maximum achievable rate $\mathrm{C} \varepsilon / \mathrm{B}$ is analyzed for MRC and $\mathrm{SC}$ combining. The $\varepsilon$ versus $\mathrm{C}_{\varepsilon} / \mathrm{B}$ tradeoff is parameterized by the turbulence-free photons per symbol $\gamma_{0}$, the normalized aperture diameter $D / r_{0}$, and the number of branches $L$ at the combiner. These information-theoretic limits quantify the optimum tradeoff between the pair $\varepsilon$ and $C_{\varepsilon} / B$, and show those set of pairs for which is impossible to find a reliable errorcorrecting codes which can realize any arbitrarily small probability of error. It is clear from Fig. 4 that, for a given probability of outage, there is a significant gain in the achievable rate when utilizing the MRC combiner over the SC combiner. sizeable additional gains in achievable rate are on hand by increasing the combiner branch number $L$, and the optimal tradeoff between $\varepsilon$ and $C_{\varepsilon} / B$ shown in Fig. 4 can be used to select code rates in feasible coherent FSO channels.

\section{CONCLUSIONS}

The noncentral chi-square distribution, which we introduced recently as a model for atmospheric fading in a single coherent receiver affected by amplitude and phase fluctuations, is used to study the performance of coherent laser communication through the turbulent atmosphere. We have compared the spectral efficiencies and SNR requirements of complex modulations in the presence of fading noise from atmospheric turbulence and local oscillator shot noise. We have discussed methods of optimizing receiver sensitivity by using atmospheric compensation techniques, including diversity combining. We have identified the impact of the combiner number of branches on the performance of coherent modulation formats. For typical turbulence conditions, sizeable gains in spectral efficiency and SNR requirements are realizable by allowing for a rather small number of apertures.

\section{ACKNOWLEDGMENT}

The research of Aniceto Belmonte was funded by the Spanish Department of Science and Technology MCYT Grant No. TEC 2006-12722

\section{REFERENCES}

[1] J. Proakis and M. Salehi, Digital Communications, (Mc Graw-Hill, 2007).

[2] D. L. Fried, "Optical heterodyne detection of an atmospherically distorted signal wave front," Proc. IEEE 55, 57-67 (1967)

[3] R. M. Gagliardi and S. Karp, Optical Communications (John Wiley \& Sons, 1995).

[4] A. Belmonte and J. M. Kahn, "Performance of synchronous optical receivers using atmospheric compensation techniques," Opt. Express 16, 14151-14162 (2008).

[5] J. D. Parsons, "Diversity techniques in communications receivers," in Advanced Signal Processing, D. A. Creasey, ed. (Peregrinus, 1985), Chap. 6.

[6] C. E. Shannon, "A mathematical theory of communications," Bell Syst. Tech. J. 27, 379-423, 623-656 (1948).

[7] A. Belmonte and J. M. Kahn, "Capacity of coherent free-space optical links using diversity-combining techniques," Opt. Express 17, 12601$12611(2009)$ 